\title{
A two-step matching algorithm for autonomous star identification GAO Yudong ${ }^{1, a}$, HUANG Senlin ${ }^{1, b}$, NIE Yao ${ }^{1, c}$
}

\author{
${ }^{1}$ College of Aerospace Science and Engineering, National University of Defense \\ Technology, Changsha 410073, China \\ agao_y_d827@sohu.com, bhuangmu2011@Yahoo.com, chieyao121@163.com
}

Keywords: two-step matching algorithm, star identification algorithm, grid algorithm, one-step matching algorithm, guide star catalog

\begin{abstract}
An algorithm based on the primary star, called the two-step matching algorithm, is proposed. The recognition characteristic of this algorithm consists of angular distances between the primary star and its neighboring stars, and the directions of neighbor stars. This algorithm uses star map information with high efficiency. The performance of the algorithm is analyzed through a simulation experiment that compares it with grid algorithm and another algorithm based on the primary star. Simulation results show that the correct identification rate of the two-step matching algorithm is more than $98 \%$ when the standard deviation of Gaussian noise is 2 pixels. This algorithm is robust with fake stars and magnitude deviation. The identification rate is more than $96 \%$ when five fake stars are present in the star maps. In addition, the algorithm has many advantages, such as quick identification, being unaffected by magnitude noise, and having a small guide star catalog. The two-step matching algorithm has good application value and is promising in the area of star identification.
\end{abstract}

\section{Introduction}

The star sensor is the most accurate attitude sensor ${ }^{[1,2]}$, and is widely used in the aerospace industry. The working processes of the star sensor include preprocessing of star maps, star point extraction, star identification, and attitude determination. Star identification is a crucial link directly related to star sensor performance. Star identification finds the unique characteristic and can distinguish different star maps. The undirected star maps are matched with the guide star catalog using the unique characteristic. The corresponding relations among stars in the field of view (FOV) of the star sensor and the guide catalog can be determined.

Numerous star identification algorithms can be broadly divided into two classes ${ }^{[3,4]}$. The first class is the subgraph isomorphism algorithms. These algorithms extract geometric characteristic using stars and the angular distance among them as vertices and edges. The formed polygons or match groups are used to perform star identification in this fashion in such algorithms. Traditional star identification algorithms, such as angular distance algorithm ${ }^{[5,6]}$, triangle algorithm ${ }^{[7-9]}$, quadrilateral algorithm ${ }^{[10]}$, and match group algorithm ${ }^{[11,12]}$, belong to this class. The second class comprises algorithms that use star 
pattern, which can be regarded as a function of geometrical characteristic. The identifying characteristic of this pattern is formed by using the function on the star that needs to be recognized and on its neighboring stars. The star being recognized is called the primary star. The aforementioned algorithms differ significantly in the construction of the identifying characteristic of primary stars. Grid algorithm ${ }^{[13]}$ and singular-value decomposition algorithm ${ }^{[14]}$ are grouped into this class of algorithm. Algorithms employing this approach are found in references ${ }^{[15-17]}$.

The identifying characteristic of the first class of algorithms is constructed from an angular distance using a relatively simple method, thus resulting in a great amount of redundancy match. The number of stars included in the identifying characteristic of the second class is usually more than the first class, and the identifying characteristic contains a higher amount of information in a more efficient method. Therefore, a small redundancy match and a high identification rate are observed.

Match group algorithm, also known as the primary star identification algorithm, was proposed by Kosik et al. and was introduced in several studies ${ }^{[11]}$. This algorithm is the earliest recognition algorithm based on the primary star. Match group algorithm belongs to the first class of algorithm. Pattern-recognition algorithms are also based on the primary star. However, the structure of the pattern is quite different. In the grid algorithm, the identification algorithm uses radial and cyclic features ${ }^{[18]}$, and is based on log-polar transform ${ }^{[19]}$. The star map is discrete to some form of grid, and the location of the stars is substituted to the location of grids in the star map. The angular distance and direction information of the star are included in the identifying characteristic. However, the substitution for the location of the stars will result in a drop in accuracy of location information. An improved star pattern identification technique based on the primary star was proposed by Wang et al. ${ }^{[20]}$. This method uses the angular distance between the primary star and its neighbors to construct the star identification characteristic. The characteristic consists of all the angular distances between the primary star and its neighbors; therefore, it is different from the match group algorithm, in which the identifying characteristic consists of only one angular distance between a pair of stars.

The identifying characteristic is the key to the star identification algorithm, and efficient characteristic extraction methods for star maps are also a major trend of development in the star identification algorithm. If more information is contained in the identifying characteristic, a greater difference among the identifying characteristics of the star maps exists. This effect is more beneficial to successful identification. Identification improvements can be proven by showing that the redundancy match of the pattern algorithm is smaller than that of the subgraph isomorphism algorithm. We propose an improved star identification algorithm based on the primary star, called the two-step matching algorithm, based on the point of view of improving information use. This algorithm proposes an efficient identifying characteristic extraction method, which results in improved star identification. 
The identifying characteristic of the two-step matching method has two sequences. The first sequence consists of an angular distance between the primary star and its neighbors; the second sequence consists of angles from a reference vector to the vectors of neighboring stars on the star map. Each sequence corresponds to one step of matching, and the first sequence amounts to the identifying characteristic employed by the algorithm of reference $^{[20]}$. The algorithm selects the reference stars after the first step of matching, thus avoiding the problem of wrong selection of reference stars that generally confronts algorithms of star pattern. The identifying characteristic consists of two sequences, namely, the undiscretization information of the angular distance and the direction of neighboring stars. Therefore, the two-step matching algorithm contains more information than the grid algorithm. A higher use of information lays the basis for a higher identification rate of the algorithm.

A two-step matching algorithm for autonomous star identification is proposed. This algorithm is more efficient for use in star map information. The performance of the two-step matching algorithm is analyzed through a simulation experiment and compared with the grid algorithm and another algorithm based on a primary star. The experiment shows that this algorithm is robust with lost stars, fake stars, and location noise. In addition, the algorithm is fast and has a small guide star catalog.

This paper is organized as follows. Section 2 introduces the basic principles of the two-step matching algorithm, including the construction of the identifying characteristic and the matching process. In Section 3, simulations are provided and the analyses and comparisons of results are discussed. Section 4 states our conclusions.

\section{The basic principles of the two-step matching algorithm}

\section{Construction of the identifying characteristic}

The process of constructing the identifying characteristic is described as follows:

1) The primary star is selected as $S$, and the neighborhood radius is supposed as $R$ ( $R$ is an angular distance). Therefore, the stars in the neighborhood of $R$ are called neighbors (assuming that a total of $N$ neighbors exist).

2) The angular distance $r_{i}$ between the $i$ th neighbor and the primary star is calculated as shown in Fig. 1. The angular distances are classified to form a sequence of angular distances in order from small to large.

$$
v_{1}=\left(r_{i}, r_{j}, r_{m} \cdots r_{k}\right), \quad \mathrm{i}, \mathrm{j}, m, \mathrm{k} \in[1, N]
$$




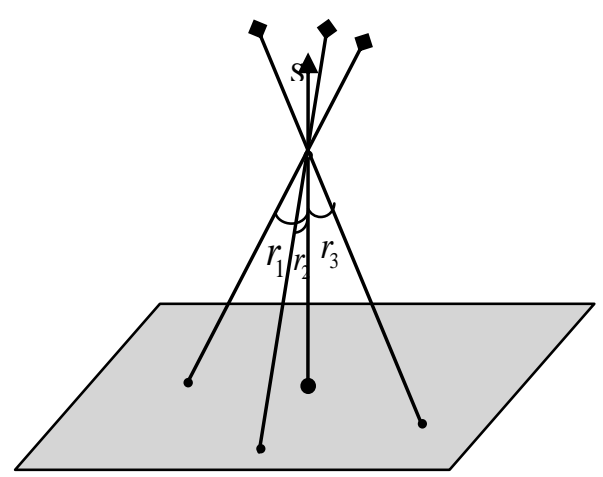

Fig. 1. The angular distances between the primary star and its neighbors.

3) The angle $\theta_{i}$ from the $\mathrm{X}$-axis positive direction to the direction of the neighbors is calculated in the primary star-centric Charge Coupled Device(CCD) plane coordinate system (clockwise) as shown in Fig. 2, and the angle sequence $v_{2}$ can be formed. Hence, $\theta_{i}$ and $r_{i}$ correspond to the same neighbor.

$$
v_{2}=\left(\theta_{i,}, \theta_{j}, \theta_{m} \cdots \theta_{k}\right)
$$

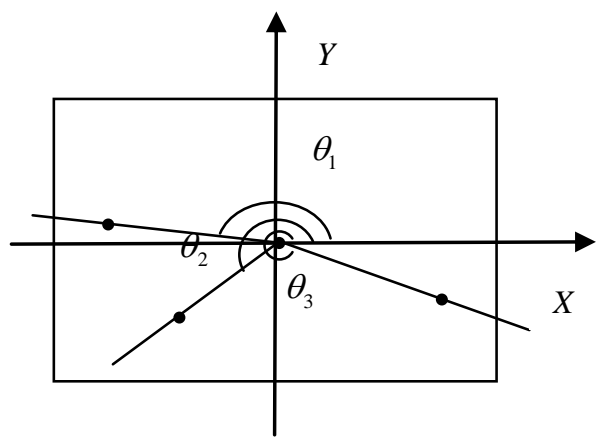

Fig. 2. Angle from the positive direction of the $\mathrm{X}$-axis to the direction of neighboring stars.

Two cases exist, namely, when the primary star is in the star map center or when it is not in the star map center. The angle from the $\mathrm{X}$-axis to the neighbor is not the same in different cases. Therefore, if the primary star is not in the star map center when sequence $v_{2}$ is calculated, then the coordinate of the star point in the CCD plane must be transformed. Stars are projected on the CCD plane once more to place the primary star on the center of the star map. The transformed star map is equivalent to a map taken by a primary-orientated star sensor.

The coordinate transformation method is expressed as follows. The coordinate of the primary star in the star map is presumed at $\left(x_{1}, y_{1}\right)$ and a neighbor is at $\left(x_{2}, y_{2}\right)$. Assuming that the focal length of the CCD is $f$, then the direction vectors of the primary star $\vec{t}_{1}$ and one of its neighbors $\vec{t}_{2}$ in the star sensor coordinate system are expressed as: 


$$
\vec{t}_{1}=-\frac{1}{\sqrt{x_{1}{ }^{2}+y_{1}{ }^{2}+f^{2}}}\left[\begin{array}{c}
x_{1} \\
y_{1} \\
-f
\end{array}\right], \quad \vec{t}_{2}=-\frac{1}{\sqrt{x_{2}{ }^{2}+y_{2}{ }^{2}+f^{2}}}\left[\begin{array}{c}
x_{2} \\
y_{2} \\
-f
\end{array}\right]
$$

$\vec{t}_{1}$ is assumed as $(\varphi, \theta, 1)$ in the spherical coordinate system. To make the Cartesian coordinates of the primary star $(0,0,1)$, the transform matrix can be obtained as follows:

$$
\mathbf{M}=\mathbf{R}_{2}(90-\theta) \mathbf{R}_{3}(\varphi)=\left[\begin{array}{ccc}
\sin \theta & 0 & -\cos \theta \\
0 & 1 & 0 \\
\cos \theta & 0 & \sin \theta
\end{array}\right] \cdot\left[\begin{array}{ccc}
\cos \varphi & \sin \varphi & 0 \\
-\sin \varphi & \cos \varphi & 0 \\
0 & 0 & 1
\end{array}\right]
$$

where $\mathbf{R}_{2}(\cdot)$ and $\mathbf{R}_{3}(\cdot)$ are elementary transform matrixes. Then, we can obtain the following expression:

$$
\left[x_{2}^{\prime}, y_{2}^{\prime}, z_{2}^{\prime}\right]^{T}=\mathbf{M} \cdot \vec{t}_{2}
$$

The coordinate of the neighboring star in the CCD image plane is given by:

$$
\left\{\begin{array}{l}
x_{2}^{\prime \prime}=-x_{2}^{\prime} \frac{f}{z_{2}^{\prime}} \\
y_{2}^{\prime \prime}=-y_{2}^{\prime} \frac{f}{z_{2}^{\prime}}
\end{array}\right.
$$

4) The identifying characteristic of the proposed algorithm is formed by $v_{1}$ and $v_{2}$. The number of neighbors in the neighborhood of the primary star is also taken as an identification condition to further improve the performance of the algorithm. The selection scope and the neighborhood radius of the primary star must be limited to make the number as accurate as possible. Then, the neighborhood of the primary star can be located as far as possible in the FOV of the star sensor. However, if the neighborhood radius of the primary star is too small, then the primary star will only have a few neighbor stars or it will have no neighbor at all. To solve this problem, the neighborhood radius of the primary star should be increased when the number of companions is less than the threshold value $T$. At the same time, the number of neighbors should not be used for matching, or the $T$ of the number should be broadened.

5) The number of neighbors of different primary stars varies, leading to different sequence lengths (the number of elements included in a sequence). This effect makes access to data difficult. Therefore, the sequence length is assumed as a normal value in this study and is expressed as $A$. The data after the Ath element in the sequence $v_{1}$ should be discarded. If the sequence length is less than $A$, then the empty seats can be filled with 0 .

\section{Matching process}

Star matching is executed in two steps after the construction of the identifying characteristic. The identifying characteristic calculated with the star maps and guide star catalog are separately denoted by $v_{i}$ and $v_{i}^{\prime}$ to facilitate the description of the matching 
process. The sequence $v_{1}$ is matched in the first step match. The matching method is shown as follows:

The elements of the two sequences are compared, which are separately presumed as $v_{1}=\left(r_{i}, r_{j}, r_{m} \cdots r_{k}\right)$ and $v_{1}^{\prime}=\left(R_{1}, R_{2}, R_{3} \cdots R_{M}\right)$. If $\left|r_{i}-R_{t}\right| \leq \delta$ is the occurrence, where $t \in[1, M]$ and $\delta$ is the match threshold, then the two elements match successfully. If the number of matched elements reaches the sequence threshold, then the two sequences match successfully.

If the first step match is successful, then the second step match is obtained. The sequence $v_{2}$ cannot be matched directly. A reference star must be selected before the second step match. The neighboring star, which has an angular distance that is the minimum value matched in sequence $v_{1}$, is selected as the reference star. Another sequence $v_{3}$ can be obtained by calculating the angle from the reference star to the other neighbors. This method can ensure the right selection of reference stars. An example of the calculation of sequence $v_{3}$ is as follows:

The minimum angular distance matched in sequence $v_{1}$ is assumed as $r_{i}$, then the following expression can be obtained:

$$
v_{3}=\left(360^{\circ}+\theta_{j}-\theta_{i}, \theta_{m}-\theta_{i} \cdots \theta_{k}-\theta_{i}, 360^{\circ}\right), \quad\left(\theta_{j}<\theta_{i}, \theta_{m}, \theta_{k}>\theta_{i}\right)
$$

The procedure for calculating sequence $v_{3}$ is shown in Fig. 3.

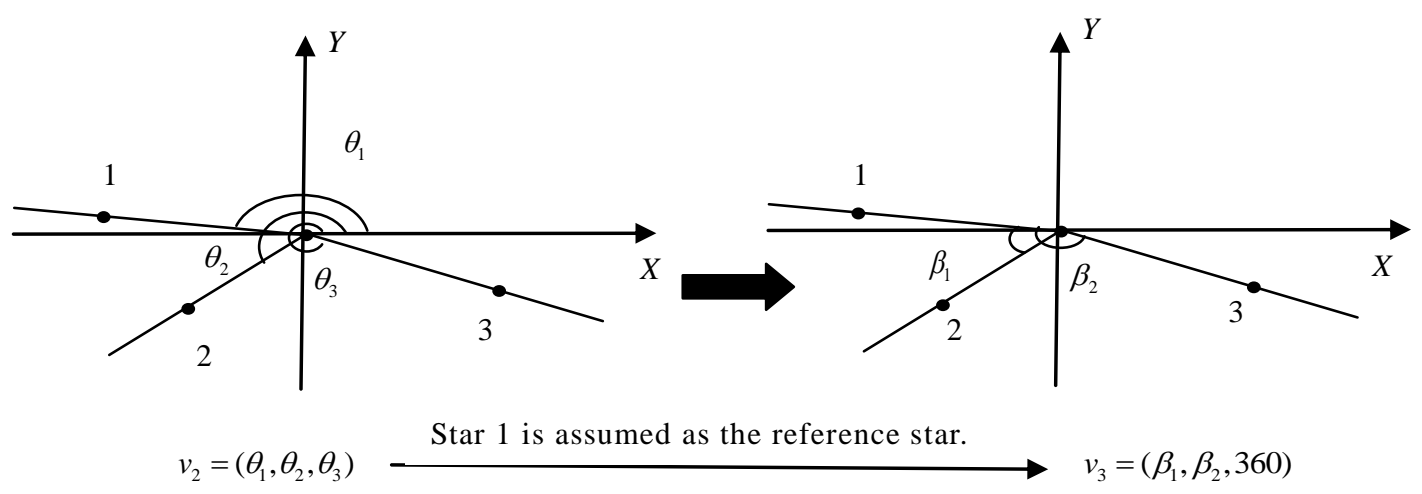

Fig. 3. Diagrammatic sketch of $v_{3}$ formed from $v_{2}$ (where $\left.\beta_{1}=\theta_{2}-\theta_{1}, \beta_{2}=\theta_{3}-\theta_{2}\right)$. $v_{3}^{\prime}$ can be calculated from $v_{1}^{\prime}$ by following the same procedure. The second step match can be performed after obtaining $v_{3}^{\prime}$. The matching method is similar to that in the first step match. The primary star of $v_{1}$ in the star map and the primary star of $v_{1}^{\prime}$ in the guide catalog match successfully when $v_{1}$ and $v_{3}$ can be matched. 
The procedure for identification of the two-step matching algorithm is shown in Fig. 4.

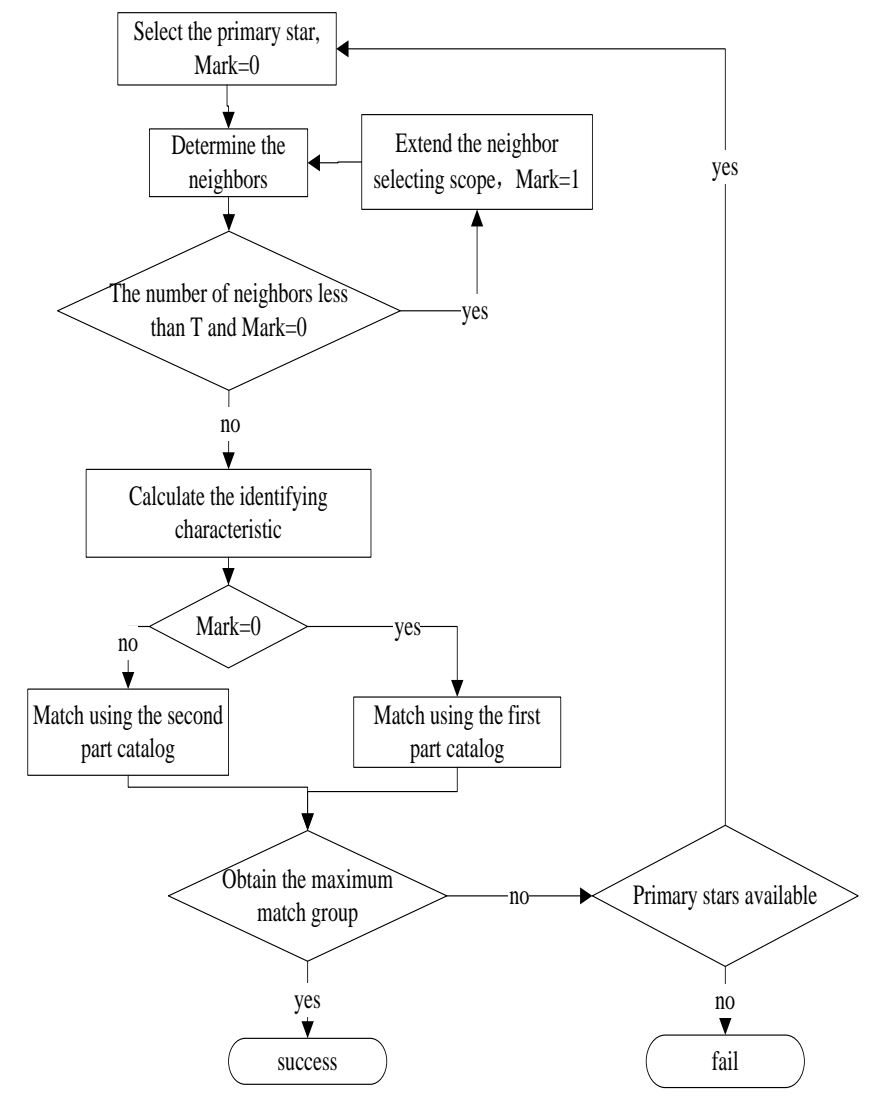

Fig. 4. Procedure for identification of the two-step matching algorithm.

\section{Simulation results and analysis}

The performance of the proposed algorithm is analyzed through a simulation experiment and compared with those of the grid algorithm and another algorithm based on the primary star. The identifying characteristic of the third algorithm only consists of angular distance, which is equivalent to the first step match in the two-step matching algorithm. This algorithm is called the one-step matching algorithm in this study. The three algorithms analyzed in the experiment are not using an index table. An index table divides the guide star catalog into several blocks according to several characteristics extracted from the identifying characteristic, such as the number of neighboring stars. The relationship between the value of these characteristics and the primary star is stored in the index table, which can be used to speed up identification. As such, the algorithm will traverse the entire guide catalog in every matching process.

\section{Parameters of experiment conditions}

1) The configuration parameters of the CCD star sensor in the simulation: $\mathrm{FOV}=14^{\circ}$ (circular field), focal length $\mathrm{f}=50 \mathrm{~mm}$. A total of 1024 pixels exist in the diameter. The size of a pixel is $0.0012 \mathrm{~mm}$.

2) Guide star catalog: The apparent magnitudes of the stars are less than six in the 
Hipparcos standard catalog. The binary stars are combined into an equivalent star. A total of 4888 stars are found in the guide star catalog. Every element of the direction vectors of the stars uses 32 bit memory, whereas the other data consume 16 bit memory.

3) Simulation platform: Core 2 2.66GHz personal computer and MATLAB R2009a platform.

4) Parameters of the algorithms:

(1) The two-step matching algorithm:

a) The neighborhood radius of the primary stars is $0.5^{\circ}<R<4^{\circ}$, and the increased neighborhood radius is $0.5^{\circ}<R<7^{\circ}$.

b) The guide star catalog consists of two parts. In part one, the neighborhood radius is $0.5^{\circ}<R<4^{\circ}$, the number of neighboring stars is more than 1 , and the number of primary stars is 4833. In the second part, the neighborhood radius is $0.5^{\circ}<R<7^{\circ}$, the number of neighboring stars is less than 5 , and the number of primary stars is 1578 . Table 1 shows an example of the storage structure of a guide star in the catalog, where the sequence length $A=12$. Every guide star uses $64 \mathrm{~B}$ memory, and the guide star catalog is $400 \mathrm{~KB}$.

Table 1. An example of the guide star catalog.

\begin{tabular}{|c|c|}
\hline Contents & Data \\
\hline 1(direction vector) & $0.9830350 .019313-0.182400$ \\
\hline 2(stellar magnitude) & 5.0 \\
\hline $\begin{array}{l}3 \text { (number } \quad \text { of } \\
\text { neighbors) }\end{array}$ & 6 \\
\hline $4\left(v_{1}\right)$ & $\begin{array}{lllllllll}1.925 & 2.568 & 2.726 & 3.248 & 3.547 & 3.673 & 0 & 0 & 0 \\
0 & 0 & 0 & & & & & & \\
\end{array}$ \\
\hline $5\left(v_{2}\right)$ & $\begin{array}{lllllllll}330.63 & 216.07 & 289.72 & 62.98 & 81.73 & 317.98 & 0 & 0 \\
0 & 0 & 0 & 0 & & & & & \\
\end{array}$ \\
\hline
\end{tabular}

(2) The structure of the catalog of the one-step matching algorithm is similar to that of the two-step matching algorithm except that the identifying characteristic of the former only consists of sequence $v_{1}$. The catalog is $250 \mathrm{~KB}$.

(3) Grid algorithm: The neighborhood radius of the primary stars is $7^{\circ}$, with a total of $60 \times 60$ grids. Each primary star uses $144 \mathrm{~B}$ memory. The catalog is $701 \mathrm{~KB}$.

\section{Experiment on magnitude noise}

To analyze the algorithm's robustness with magnitude noise, Gaussian noise is added in the simulation star maps, in which the mean value is 0 and standard deviation ranges from 0 to 2 . The statistical data of identification of 5000 star maps are generated randomly from a full-sky. The identification rate exactly represents a successful identification rate as shown in Fig. 5. According to Fig. 5, the three algorithms are almost unaffected by magnitude noise because magnitude is not used as an identifying characteristic in the algorithms. 


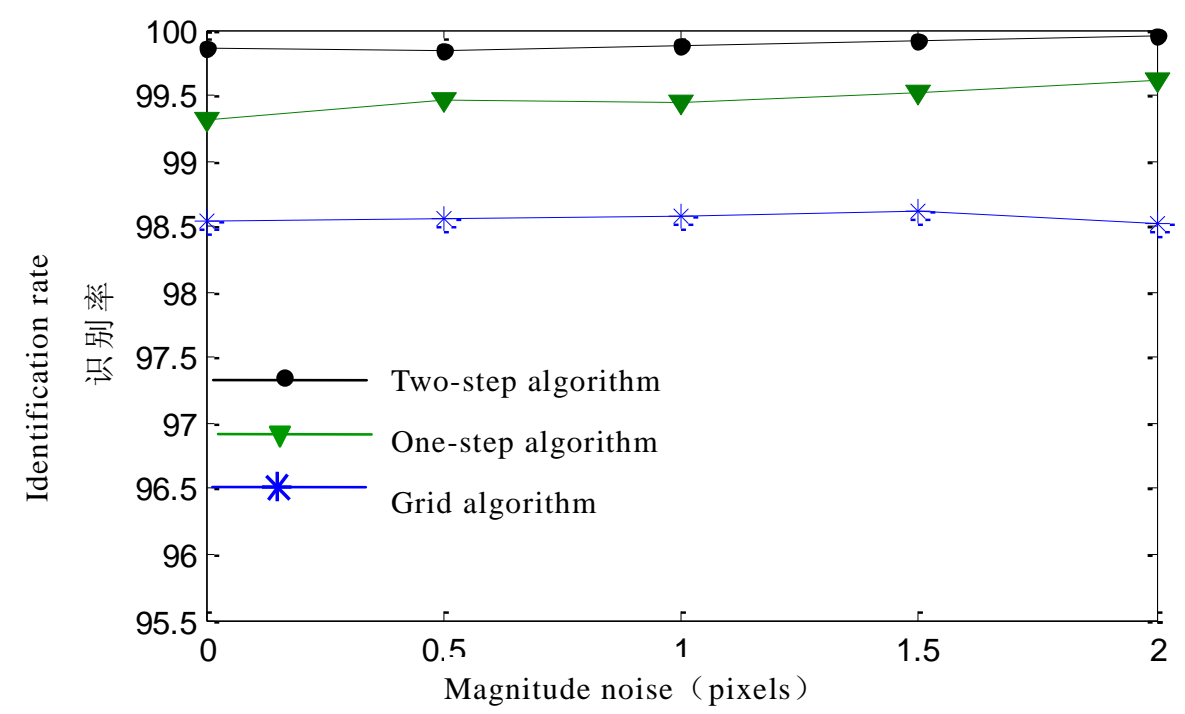

Fig. 5. Identification rate versus magnitude noise.

\section{Experiment on location noise}

To analyze the robustness of the algorithm with location noise, Gaussian noise is added in the simulation star maps, in which the mean value is 0 and standard deviation ranges from 0 to 2 . The statistical data of the identification rate of 5000 star maps are generated randomly from a full-sky. The result shows that the identification rate of the two-step matching algorithm is higher than $98 \%$ when Gaussian noise is 2 pixels (Fig. 6). The identification rate of the two-step matching algorithm is higher than those of the other two algorithms.

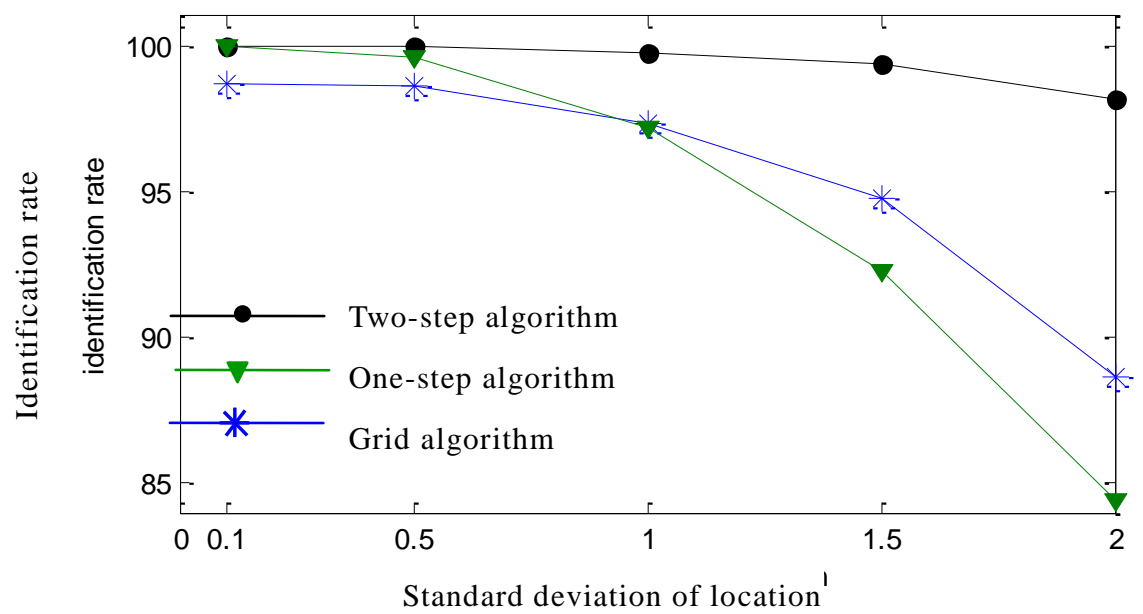

Fig. 6. Identification rate versus location accuracy.

\section{Experiment on lost stars}

A lost star exists in the guide star catalogue but it cannot be found in the star map. To analyze the robustness of the algorithm with lost stars, one to five stars are removed from the simulation star maps. The statistical data of identification of 5000 star maps are generated randomly from a full-sky. The two-step matching algorithm uses more accurate information than the other two algorithms (Fig. 7), therefore, it needs fewer stars to make a 
successful identification. The two-step matching algorithm is more robust against lost stars. When five lost stars exist in the field, the identification rate of the two-step matching algorithm is $97 \%$, whereas that of the grid algorithm is $84 \%$, and that of the one-step matching algorithm is $87 \%$.

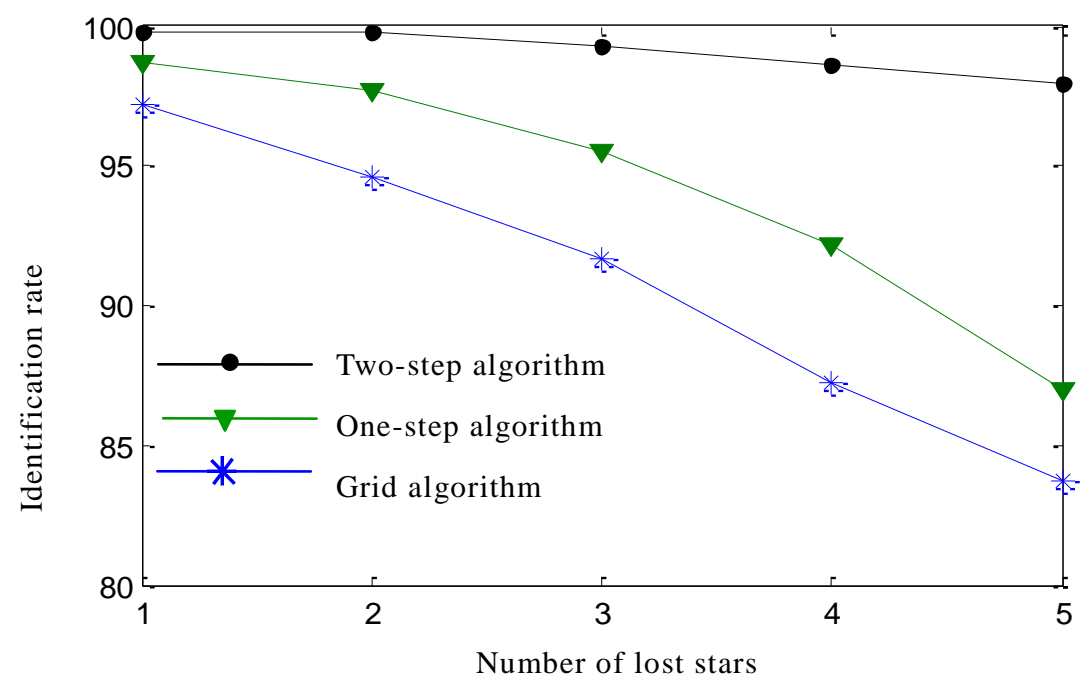

Fig. 7. Identification rate versus lost stars.

\section{Experiment on fake stars}

To analyze the robustness of the algorithm with fake stars, one to five fake stars are added in the simulation star maps. The statistical data of identification of 5000 star maps are generated randomly from a full-sky. The result shows that the two-step matching algorithm is almost unaffected by fake stars (Fig. 8) when the number of fake stars is less than three. Furthermore, the identification rate is higher than $96 \%$ when five fake stars are present in the field. The identification rate of the two-step matching algorithm is higher than those of the other two algorithms.

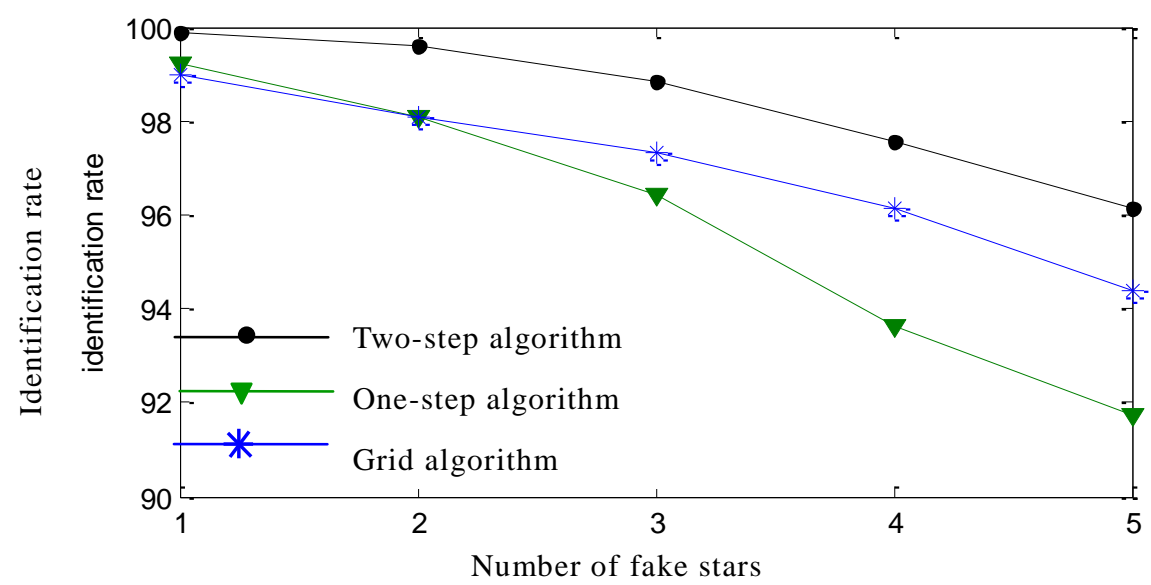

Fig. 8. Identification rate versus fake stars.

\section{Time consumed}

Table 2 shows the mean consumed time of the three algorithms at different noise levels. Each algorithm identifies 5000 star maps and the unit of time is ms. We can see from the table that the two-step matching algorithm is faster than the grid algorithm, and is 
the most unaffected by noise.

Table 1. Comparison of time consumed.

\begin{tabular}{|c|c|c|c|}
\hline Noise (pixel) & $\begin{array}{c}\text { Two-step } \\
(\mathrm{ms})\end{array}$ & $\begin{array}{c}\text { One-step } \\
(\mathrm{ms})\end{array}$ & $\begin{array}{c}\text { Grid } \\
(\mathrm{ms})\end{array}$ \\
\hline 0.1 & 23 & 11 & 119 \\
\hline 1 & 30 & 33 & 355 \\
\hline 2 & 43 & 48 & 660 \\
\hline
\end{tabular}

\section{Conclusions}

A new star identification algorithm based on the primary star, called the two-step matching algorithm, is designed in this paper. Compared with traditional identification algorithms based on the primary star, the proposed algorithm does not only use the angular distance between the primary star and its neighbors, but also the angle between the direction vectors of neighboring stars. The information use efficiency of the algorithm is twice than that of the traditional algorithm based on the primary star, leading to a high identification rate. The problem of wrong selection of reference stars is avoided in the two-step matching algorithm. The performance of the two-step matching algorithm is analyzed through a simulation experiment and compared with those of the grid algorithm and the one-step matching algorithm. The experiment results show that the identification rate of the two-step matching algorithm is more than $98 \%$ when the standard deviation of Gaussian noise is 2 pixels. The proposed algorithm is robust against fake stars and magnitude deviation. The identification rate is still more than $96 \%$ when five fake stars are present in the star maps. In addition, the two-step matching algorithm is unaffected by magnitude noise because magnitude is not used in identification. In addition, this algorithm has a small guide catalog and the time it consumes is less. Therefore, the two-step matching algorithm has good applications and a promising future.

\section{References}

[1] Liebe C C. Accuracy performance of star trackers. IEEE Transaction on aerospace and electronic systems, 2002;38(2):591-592.

[2] Wang X D. Study on wild-field-of-view and high-accuracy star sensor technologies. Ph.D. Dissertation, Chang chun Institute of Optics, Fine Mechanics and Physics Academia Sinica,2003.[in Chinese]

[3] Padgett C, Kdeigado K, Udomkesmalee S. Evaluation of star identification techniques. Journal of Guidance, Control and Dynamics, 1997;20(2):259-267.

[4] Zhang G J. Star identification. Beijing: National defense industry press,2011. [in Chinese]

[5] Cole C L, Crassidis J L. Fast star pattern recognition using planar triangles. Journal of 
Guidance, Control and Dynamics, 2006; 29(1):64-71.

[6] Gottlieb D M. Star identification techniques, Spacecraft Attitude Determination and Control, Edited by J. R. Werz, The Netherlands, 1978; 257-266.

[7] Liebe C C. Star trackers for attitude determination. IEEE Transaction on Aerospace and Electronic system, 1995;10(6):10-16.

[8] Li L H, Lin T, Ning Y C, et al. Improved all-sky autonomous triangle star-field identification algorithm. Optical Technique, 2000;26(4):372-374. [in Chinese]

[9] Tabur V. Fast algorithms for matching CCD images to a stellar catalogue. Publications of the Astronomical Society of Australia, 2007;24(4):189-198.

[10]Lin T, Zhou J L, Zhang J P, et al. All-sky automated quaternary star pattern recognition. Journal of astronautics, 2000;21(2):82-85. [in Chinese]

[11]Kosik J. Star pattern identification aboard an inertially stabilized spacecraft. Journal of Guidance, Control and Dynamics, 1991;14(1):230-235.

[12]Bezooijen R V. Automated star pattern recognition. Ph.D. Dissertation, Stanford University, 1989.

[13]Padgett C, KREUTZ-DELGADO K. A grid algorithm for autonomous star identification. IEEE Transaction on aerospace and electronic systems, 1997;33(1):202-213.

[14]Zhu C Z. Star pattern identification algorithms and attitude determination techniques based on star sensor, Ph.D. Dissertation, National University of defense technology,2004. [in Chinese]

[15]Liebe C C. Pattern recognition of star constellations for spacecraft applications. IEEE Aeronautics and Electronic Systems Magazine. 1992;(10):2-12.

[16]Hyunjae L, Hyochoong B. Star pattern identification technique by modified grid algorithm. IEEE Transactions on Aerospace and Electronic Systems, 2007;43(3):1112-1116.

[17]Ho K. A survey of algorithms for star identification with low-cost star trackers. Acta Astronautica, 2012;(73):156-163.

[18]Zhang G J, Wei X G, Jiang J. Full-sky autonomous star identification based on radial and cyclic features of star pattern. Image and vision computing, 2008;26(7):891-897. [in Chinese]

[19]Zhang G J, Wei X G, Jiang J. Star identification algorithm based on Log-Polar transform. Journal of Aerospace Computing Information and Communication, 2009;6(8):483-490. [in Chinese]

[20]Wang H Y, Fei Z H, Zhang C. An improved star pattern identification algorithm based on main star. Optics and Precision Engineering, 2009;17(1):220-224. [in Chinese] 